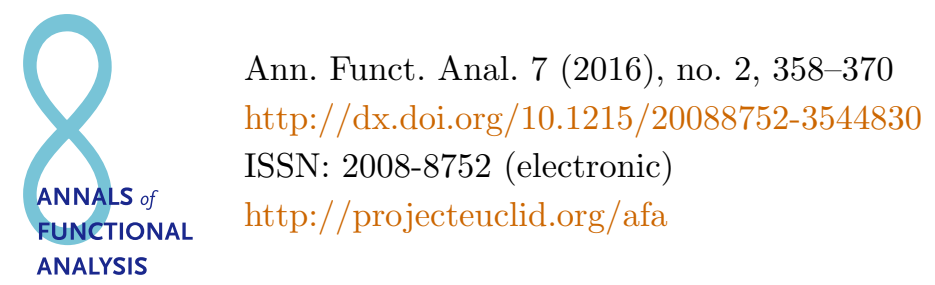

\title{
SCALE TRANSFORMATIONS FOR PRESENT POSITION-DEPENDENT CONDITIONAL EXPECTATIONS OVER CONTINUOUS PATHS
}

\author{
DONG HYUN CHO
}

Communicated by S. Barza

Abstract. Let $C[0, t]$ denote a generalized Wiener space, the space of realvalued continuous functions on the interval $[0, t]$, and define a random vector $Z_{n}: C[0, t] \rightarrow \mathbb{R}^{n}$ by

$$
Z_{n}(x)=\left(\int_{0}^{t_{1}} h(s) d x(s), \ldots, \int_{0}^{t_{n}} h(s) d x(s)\right)
$$

where $0<t_{1}<\cdots<t_{n}=t$ is a partition of $[0, t]$ and $h \in L_{2}[0, t]$ with $h \neq 0$ almost everywhere. Using a simple formula for a generalized conditional Wiener integral on $C[0, t]$ with the conditioning function $Z_{n}$, we evaluate the generalized analytic conditional Wiener and Feynman integrals of the cylinder function

$$
G(x)=f((e, x)) \phi((e, x))
$$

for $x \in C[0, t]$, where $f \in L_{p}(\mathbb{R})(1 \leq p \leq \infty)$, $e$ is a unit element in $L_{2}[0, t]$, and $\phi$ is the Fourier transform of a measure of bounded variation over $\mathbb{R}$. We then express the generalized analytic conditional Feynman integral of $G$ as two kinds of limits of nonconditional generalized Wiener integrals with a polygonal function and cylinder functions using a change-of-scale transformation. The choice of a complete orthonormal subset of $L_{2}[0, t]$ used in the transformation is independent of $e$.

Copyright 2016 by the Tusi Mathematical Research Group.

Received Aug. 25, 2015; Accepted Nov. 2, 2015.

2010 Mathematics Subject Classification. Primary 46T12; Secondary 28C20, 46 G12.

Keywords. analytic conditional Feynman integral, analytic conditional Wiener integral, conditional Wiener integral, Wiener integral, Wiener space. 


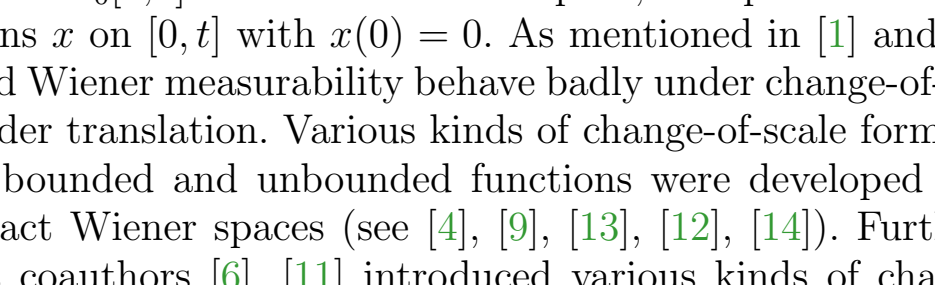

\title{
SCALE TRANSFORMATIONS FOR PRESENT POSITION-DEPENDENT CONDITIONAL EXPECTATIONS OVER CONTINUOUS PATHS
}

\author{
DONG HYUN CHO
}

Communicated by S. Barza

Abstract. Let $C[0, t]$ denote a generalized Wiener space, the space of realvalued continuous functions on the interval $[0, t]$, and define a random vector $Z_{n}: C[0, t] \rightarrow \mathbb{R}^{n}$ by

$$
Z_{n}(x)=\left(\int_{0}^{t_{1}} h(s) d x(s), \ldots, \int_{0}^{t_{n}} h(s) d x(s)\right)
$$

where $0<t_{1}<\cdots<t_{n}=t$ is a partition of $[0, t]$ and $h \in L_{2}[0, t]$ with $h \neq 0$ almost everywhere. Using a simple formula for a generalized conditional Wiener integral on $C[0, t]$ with the conditioning function $Z_{n}$, we evaluate the generalized analytic conditional Wiener and Feynman integrals of the cylinder function

$$
G(x)=f((e, x)) \phi((e, x))
$$

for $x \in C[0, t]$, where $f \in L_{p}(\mathbb{R})(1 \leq p \leq \infty)$, $e$ is a unit element in $L_{2}[0, t]$, and $\phi$ is the Fourier transform of a measure of bounded variation over $\mathbb{R}$. We then express the generalized analytic conditional Feynman integral of $G$ as two kinds of limits of nonconditional generalized Wiener integrals with a polygonal function and cylinder functions using a change-of-scale transformation. The choice of a complete orthonormal subset of $L_{2}[0, t]$ used in the transformation is independent of $e$.

Copyright 2016 by the Tusi Mathematical Research Group.

Received Aug. 25, 2015; Accepted Nov. 2, 2015.

2010 Mathematics Subject Classification. Primary 46T12; Secondary 28C20, 46 G12.

Keywords. analytic conditional Feynman integral, analytic conditional Wiener integral, conditional Wiener integral, Wiener integral, Wiener space. 


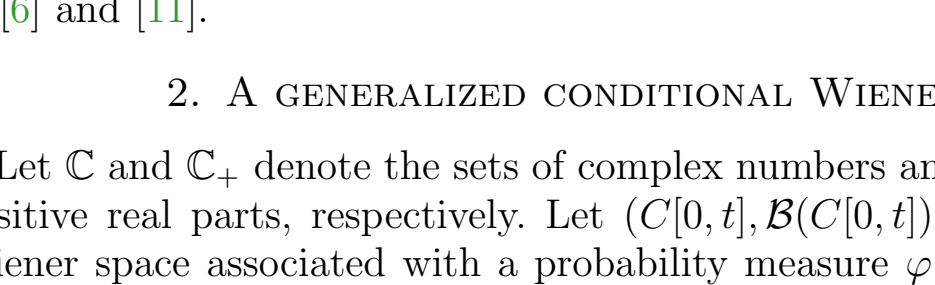

\title{
SCALE TRANSFORMATIONS FOR PRESENT POSITION-DEPENDENT CONDITIONAL EXPECTATIONS OVER CONTINUOUS PATHS
}

\author{
DONG HYUN CHO
}

Communicated by S. Barza

Abstract. Let $C[0, t]$ denote a generalized Wiener space, the space of realvalued continuous functions on the interval $[0, t]$, and define a random vector $Z_{n}: C[0, t] \rightarrow \mathbb{R}^{n}$ by

$$
Z_{n}(x)=\left(\int_{0}^{t_{1}} h(s) d x(s), \ldots, \int_{0}^{t_{n}} h(s) d x(s)\right)
$$

where $0<t_{1}<\cdots<t_{n}=t$ is a partition of $[0, t]$ and $h \in L_{2}[0, t]$ with $h \neq 0$ almost everywhere. Using a simple formula for a generalized conditional Wiener integral on $C[0, t]$ with the conditioning function $Z_{n}$, we evaluate the generalized analytic conditional Wiener and Feynman integrals of the cylinder function

$$
G(x)=f((e, x)) \phi((e, x))
$$

for $x \in C[0, t]$, where $f \in L_{p}(\mathbb{R})(1 \leq p \leq \infty)$, $e$ is a unit element in $L_{2}[0, t]$, and $\phi$ is the Fourier transform of a measure of bounded variation over $\mathbb{R}$. We then express the generalized analytic conditional Feynman integral of $G$ as two kinds of limits of nonconditional generalized Wiener integrals with a polygonal function and cylinder functions using a change-of-scale transformation. The choice of a complete orthonormal subset of $L_{2}[0, t]$ used in the transformation is independent of $e$.

Copyright 2016 by the Tusi Mathematical Research Group.

Received Aug. 25, 2015; Accepted Nov. 2, 2015.

2010 Mathematics Subject Classification. Primary 46T12; Secondary 28C20, 46 G12.

Keywords. analytic conditional Feynman integral, analytic conditional Wiener integral, conditional Wiener integral, Wiener integral, Wiener space. 


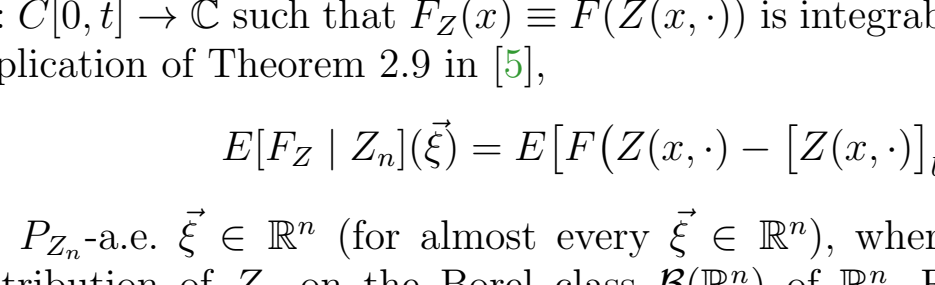

\title{
SCALE TRANSFORMATIONS FOR PRESENT POSITION-DEPENDENT CONDITIONAL EXPECTATIONS OVER CONTINUOUS PATHS
}

\author{
DONG HYUN CHO
}

Communicated by S. Barza

Abstract. Let $C[0, t]$ denote a generalized Wiener space, the space of realvalued continuous functions on the interval $[0, t]$, and define a random vector $Z_{n}: C[0, t] \rightarrow \mathbb{R}^{n}$ by

$$
Z_{n}(x)=\left(\int_{0}^{t_{1}} h(s) d x(s), \ldots, \int_{0}^{t_{n}} h(s) d x(s)\right)
$$

where $0<t_{1}<\cdots<t_{n}=t$ is a partition of $[0, t]$ and $h \in L_{2}[0, t]$ with $h \neq 0$ almost everywhere. Using a simple formula for a generalized conditional Wiener integral on $C[0, t]$ with the conditioning function $Z_{n}$, we evaluate the generalized analytic conditional Wiener and Feynman integrals of the cylinder function

$$
G(x)=f((e, x)) \phi((e, x))
$$

for $x \in C[0, t]$, where $f \in L_{p}(\mathbb{R})(1 \leq p \leq \infty)$, $e$ is a unit element in $L_{2}[0, t]$, and $\phi$ is the Fourier transform of a measure of bounded variation over $\mathbb{R}$. We then express the generalized analytic conditional Feynman integral of $G$ as two kinds of limits of nonconditional generalized Wiener integrals with a polygonal function and cylinder functions using a change-of-scale transformation. The choice of a complete orthonormal subset of $L_{2}[0, t]$ used in the transformation is independent of $e$.

Copyright 2016 by the Tusi Mathematical Research Group.

Received Aug. 25, 2015; Accepted Nov. 2, 2015.

2010 Mathematics Subject Classification. Primary 46T12; Secondary 28C20, 46 G12.

Keywords. analytic conditional Feynman integral, analytic conditional Wiener integral, conditional Wiener integral, Wiener integral, Wiener space. 


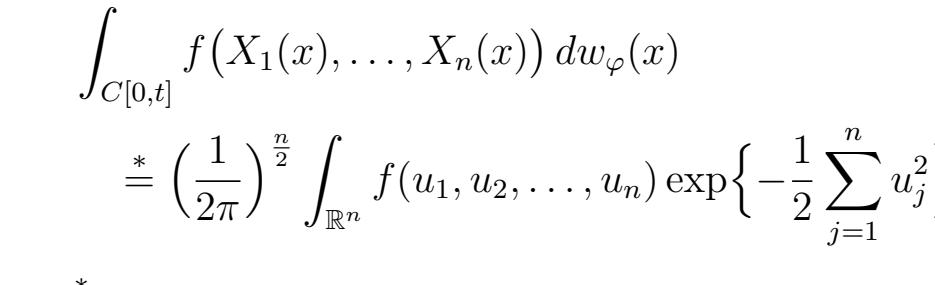

\title{
SCALE TRANSFORMATIONS FOR PRESENT POSITION-DEPENDENT CONDITIONAL EXPECTATIONS OVER CONTINUOUS PATHS
}

\author{
DONG HYUN CHO
}

Communicated by S. Barza

Abstract. Let $C[0, t]$ denote a generalized Wiener space, the space of realvalued continuous functions on the interval $[0, t]$, and define a random vector $Z_{n}: C[0, t] \rightarrow \mathbb{R}^{n}$ by

$$
Z_{n}(x)=\left(\int_{0}^{t_{1}} h(s) d x(s), \ldots, \int_{0}^{t_{n}} h(s) d x(s)\right)
$$

where $0<t_{1}<\cdots<t_{n}=t$ is a partition of $[0, t]$ and $h \in L_{2}[0, t]$ with $h \neq 0$ almost everywhere. Using a simple formula for a generalized conditional Wiener integral on $C[0, t]$ with the conditioning function $Z_{n}$, we evaluate the generalized analytic conditional Wiener and Feynman integrals of the cylinder function

$$
G(x)=f((e, x)) \phi((e, x))
$$

for $x \in C[0, t]$, where $f \in L_{p}(\mathbb{R})(1 \leq p \leq \infty)$, $e$ is a unit element in $L_{2}[0, t]$, and $\phi$ is the Fourier transform of a measure of bounded variation over $\mathbb{R}$. We then express the generalized analytic conditional Feynman integral of $G$ as two kinds of limits of nonconditional generalized Wiener integrals with a polygonal function and cylinder functions using a change-of-scale transformation. The choice of a complete orthonormal subset of $L_{2}[0, t]$ used in the transformation is independent of $e$.

Copyright 2016 by the Tusi Mathematical Research Group.

Received Aug. 25, 2015; Accepted Nov. 2, 2015.

2010 Mathematics Subject Classification. Primary 46T12; Secondary 28C20, 46 G12.

Keywords. analytic conditional Feynman integral, analytic conditional Wiener integral, conditional Wiener integral, Wiener integral, Wiener space. 


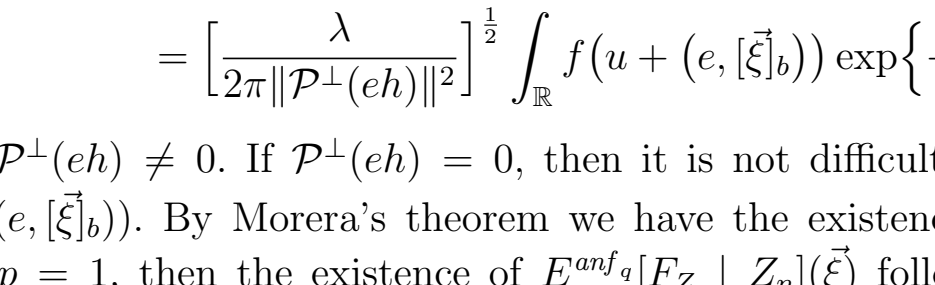

\title{
SCALE TRANSFORMATIONS FOR PRESENT POSITION-DEPENDENT CONDITIONAL EXPECTATIONS OVER CONTINUOUS PATHS
}

\author{
DONG HYUN CHO
}

Communicated by S. Barza

Abstract. Let $C[0, t]$ denote a generalized Wiener space, the space of realvalued continuous functions on the interval $[0, t]$, and define a random vector $Z_{n}: C[0, t] \rightarrow \mathbb{R}^{n}$ by

$$
Z_{n}(x)=\left(\int_{0}^{t_{1}} h(s) d x(s), \ldots, \int_{0}^{t_{n}} h(s) d x(s)\right)
$$

where $0<t_{1}<\cdots<t_{n}=t$ is a partition of $[0, t]$ and $h \in L_{2}[0, t]$ with $h \neq 0$ almost everywhere. Using a simple formula for a generalized conditional Wiener integral on $C[0, t]$ with the conditioning function $Z_{n}$, we evaluate the generalized analytic conditional Wiener and Feynman integrals of the cylinder function

$$
G(x)=f((e, x)) \phi((e, x))
$$

for $x \in C[0, t]$, where $f \in L_{p}(\mathbb{R})(1 \leq p \leq \infty)$, $e$ is a unit element in $L_{2}[0, t]$, and $\phi$ is the Fourier transform of a measure of bounded variation over $\mathbb{R}$. We then express the generalized analytic conditional Feynman integral of $G$ as two kinds of limits of nonconditional generalized Wiener integrals with a polygonal function and cylinder functions using a change-of-scale transformation. The choice of a complete orthonormal subset of $L_{2}[0, t]$ used in the transformation is independent of $e$.

Copyright 2016 by the Tusi Mathematical Research Group.

Received Aug. 25, 2015; Accepted Nov. 2, 2015.

2010 Mathematics Subject Classification. Primary 46T12; Secondary 28C20, 46 G12.

Keywords. analytic conditional Feynman integral, analytic conditional Wiener integral, conditional Wiener integral, Wiener integral, Wiener space. 


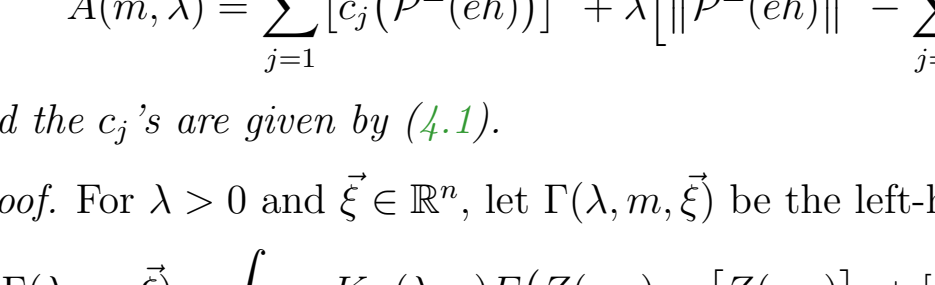

\title{
SCALE TRANSFORMATIONS FOR PRESENT POSITION-DEPENDENT CONDITIONAL EXPECTATIONS OVER CONTINUOUS PATHS
}

\author{
DONG HYUN CHO
}

Communicated by S. Barza

Abstract. Let $C[0, t]$ denote a generalized Wiener space, the space of realvalued continuous functions on the interval $[0, t]$, and define a random vector $Z_{n}: C[0, t] \rightarrow \mathbb{R}^{n}$ by

$$
Z_{n}(x)=\left(\int_{0}^{t_{1}} h(s) d x(s), \ldots, \int_{0}^{t_{n}} h(s) d x(s)\right)
$$

where $0<t_{1}<\cdots<t_{n}=t$ is a partition of $[0, t]$ and $h \in L_{2}[0, t]$ with $h \neq 0$ almost everywhere. Using a simple formula for a generalized conditional Wiener integral on $C[0, t]$ with the conditioning function $Z_{n}$, we evaluate the generalized analytic conditional Wiener and Feynman integrals of the cylinder function

$$
G(x)=f((e, x)) \phi((e, x))
$$

for $x \in C[0, t]$, where $f \in L_{p}(\mathbb{R})(1 \leq p \leq \infty)$, $e$ is a unit element in $L_{2}[0, t]$, and $\phi$ is the Fourier transform of a measure of bounded variation over $\mathbb{R}$. We then express the generalized analytic conditional Feynman integral of $G$ as two kinds of limits of nonconditional generalized Wiener integrals with a polygonal function and cylinder functions using a change-of-scale transformation. The choice of a complete orthonormal subset of $L_{2}[0, t]$ used in the transformation is independent of $e$.

Copyright 2016 by the Tusi Mathematical Research Group.

Received Aug. 25, 2015; Accepted Nov. 2, 2015.

2010 Mathematics Subject Classification. Primary 46T12; Secondary 28C20, 46 G12.

Keywords. analytic conditional Feynman integral, analytic conditional Wiener integral, conditional Wiener integral, Wiener integral, Wiener space. 


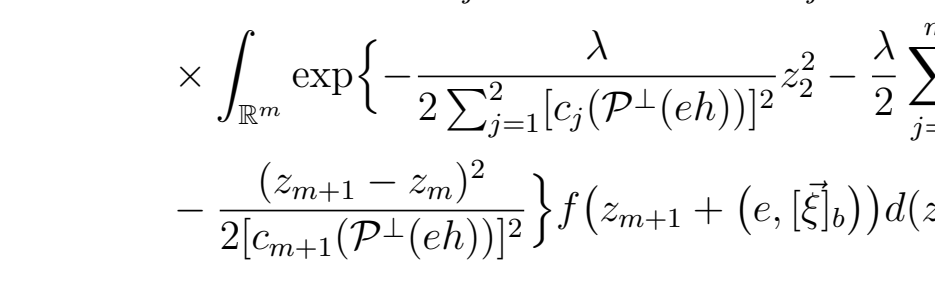

\title{
SCALE TRANSFORMATIONS FOR PRESENT POSITION-DEPENDENT CONDITIONAL EXPECTATIONS OVER CONTINUOUS PATHS
}

\author{
DONG HYUN CHO
}

Communicated by S. Barza

Abstract. Let $C[0, t]$ denote a generalized Wiener space, the space of realvalued continuous functions on the interval $[0, t]$, and define a random vector $Z_{n}: C[0, t] \rightarrow \mathbb{R}^{n}$ by

$$
Z_{n}(x)=\left(\int_{0}^{t_{1}} h(s) d x(s), \ldots, \int_{0}^{t_{n}} h(s) d x(s)\right)
$$

where $0<t_{1}<\cdots<t_{n}=t$ is a partition of $[0, t]$ and $h \in L_{2}[0, t]$ with $h \neq 0$ almost everywhere. Using a simple formula for a generalized conditional Wiener integral on $C[0, t]$ with the conditioning function $Z_{n}$, we evaluate the generalized analytic conditional Wiener and Feynman integrals of the cylinder function

$$
G(x)=f((e, x)) \phi((e, x))
$$

for $x \in C[0, t]$, where $f \in L_{p}(\mathbb{R})(1 \leq p \leq \infty)$, $e$ is a unit element in $L_{2}[0, t]$, and $\phi$ is the Fourier transform of a measure of bounded variation over $\mathbb{R}$. We then express the generalized analytic conditional Feynman integral of $G$ as two kinds of limits of nonconditional generalized Wiener integrals with a polygonal function and cylinder functions using a change-of-scale transformation. The choice of a complete orthonormal subset of $L_{2}[0, t]$ used in the transformation is independent of $e$.

Copyright 2016 by the Tusi Mathematical Research Group.

Received Aug. 25, 2015; Accepted Nov. 2, 2015.

2010 Mathematics Subject Classification. Primary 46T12; Secondary 28C20, 46 G12.

Keywords. analytic conditional Feynman integral, analytic conditional Wiener integral, conditional Wiener integral, Wiener integral, Wiener space. 


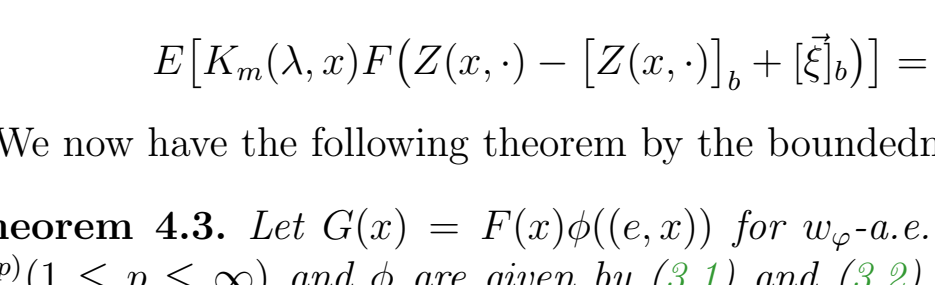

\title{
SCALE TRANSFORMATIONS FOR PRESENT POSITION-DEPENDENT CONDITIONAL EXPECTATIONS OVER CONTINUOUS PATHS
}

\author{
DONG HYUN CHO
}

Communicated by S. Barza

Abstract. Let $C[0, t]$ denote a generalized Wiener space, the space of realvalued continuous functions on the interval $[0, t]$, and define a random vector $Z_{n}: C[0, t] \rightarrow \mathbb{R}^{n}$ by

$$
Z_{n}(x)=\left(\int_{0}^{t_{1}} h(s) d x(s), \ldots, \int_{0}^{t_{n}} h(s) d x(s)\right)
$$

where $0<t_{1}<\cdots<t_{n}=t$ is a partition of $[0, t]$ and $h \in L_{2}[0, t]$ with $h \neq 0$ almost everywhere. Using a simple formula for a generalized conditional Wiener integral on $C[0, t]$ with the conditioning function $Z_{n}$, we evaluate the generalized analytic conditional Wiener and Feynman integrals of the cylinder function

$$
G(x)=f((e, x)) \phi((e, x))
$$

for $x \in C[0, t]$, where $f \in L_{p}(\mathbb{R})(1 \leq p \leq \infty)$, $e$ is a unit element in $L_{2}[0, t]$, and $\phi$ is the Fourier transform of a measure of bounded variation over $\mathbb{R}$. We then express the generalized analytic conditional Feynman integral of $G$ as two kinds of limits of nonconditional generalized Wiener integrals with a polygonal function and cylinder functions using a change-of-scale transformation. The choice of a complete orthonormal subset of $L_{2}[0, t]$ used in the transformation is independent of $e$.

Copyright 2016 by the Tusi Mathematical Research Group.

Received Aug. 25, 2015; Accepted Nov. 2, 2015.

2010 Mathematics Subject Classification. Primary 46T12; Secondary 28C20, 46 G12.

Keywords. analytic conditional Feynman integral, analytic conditional Wiener integral, conditional Wiener integral, Wiener integral, Wiener space. 


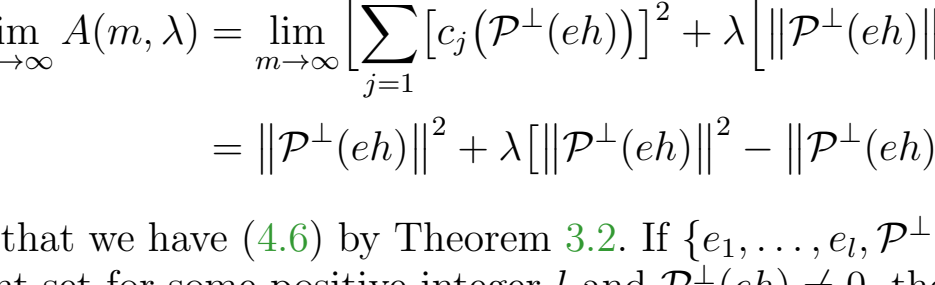

\title{
SCALE TRANSFORMATIONS FOR PRESENT POSITION-DEPENDENT CONDITIONAL EXPECTATIONS OVER CONTINUOUS PATHS
}

\author{
DONG HYUN CHO
}

Communicated by S. Barza

Abstract. Let $C[0, t]$ denote a generalized Wiener space, the space of realvalued continuous functions on the interval $[0, t]$, and define a random vector $Z_{n}: C[0, t] \rightarrow \mathbb{R}^{n}$ by

$$
Z_{n}(x)=\left(\int_{0}^{t_{1}} h(s) d x(s), \ldots, \int_{0}^{t_{n}} h(s) d x(s)\right)
$$

where $0<t_{1}<\cdots<t_{n}=t$ is a partition of $[0, t]$ and $h \in L_{2}[0, t]$ with $h \neq 0$ almost everywhere. Using a simple formula for a generalized conditional Wiener integral on $C[0, t]$ with the conditioning function $Z_{n}$, we evaluate the generalized analytic conditional Wiener and Feynman integrals of the cylinder function

$$
G(x)=f((e, x)) \phi((e, x))
$$

for $x \in C[0, t]$, where $f \in L_{p}(\mathbb{R})(1 \leq p \leq \infty)$, $e$ is a unit element in $L_{2}[0, t]$, and $\phi$ is the Fourier transform of a measure of bounded variation over $\mathbb{R}$. We then express the generalized analytic conditional Feynman integral of $G$ as two kinds of limits of nonconditional generalized Wiener integrals with a polygonal function and cylinder functions using a change-of-scale transformation. The choice of a complete orthonormal subset of $L_{2}[0, t]$ used in the transformation is independent of $e$.

Copyright 2016 by the Tusi Mathematical Research Group.

Received Aug. 25, 2015; Accepted Nov. 2, 2015.

2010 Mathematics Subject Classification. Primary 46T12; Secondary 28C20, 46 G12.

Keywords. analytic conditional Feynman integral, analytic conditional Wiener integral, conditional Wiener integral, Wiener integral, Wiener space. 


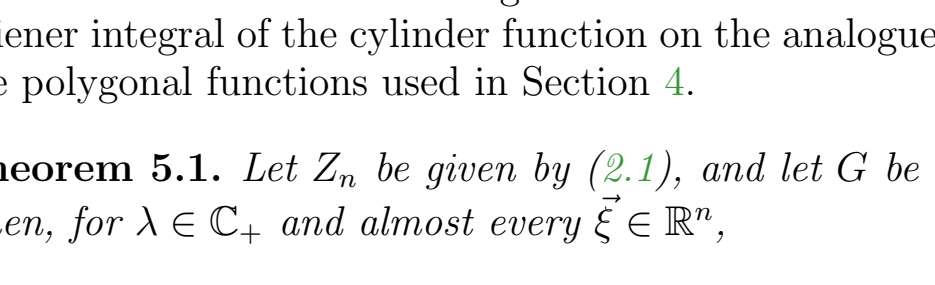

\title{
SCALE TRANSFORMATIONS FOR PRESENT POSITION-DEPENDENT CONDITIONAL EXPECTATIONS OVER CONTINUOUS PATHS
}

\author{
DONG HYUN CHO
}

Communicated by S. Barza

Abstract. Let $C[0, t]$ denote a generalized Wiener space, the space of realvalued continuous functions on the interval $[0, t]$, and define a random vector $Z_{n}: C[0, t] \rightarrow \mathbb{R}^{n}$ by

$$
Z_{n}(x)=\left(\int_{0}^{t_{1}} h(s) d x(s), \ldots, \int_{0}^{t_{n}} h(s) d x(s)\right)
$$

where $0<t_{1}<\cdots<t_{n}=t$ is a partition of $[0, t]$ and $h \in L_{2}[0, t]$ with $h \neq 0$ almost everywhere. Using a simple formula for a generalized conditional Wiener integral on $C[0, t]$ with the conditioning function $Z_{n}$, we evaluate the generalized analytic conditional Wiener and Feynman integrals of the cylinder function

$$
G(x)=f((e, x)) \phi((e, x))
$$

for $x \in C[0, t]$, where $f \in L_{p}(\mathbb{R})(1 \leq p \leq \infty)$, $e$ is a unit element in $L_{2}[0, t]$, and $\phi$ is the Fourier transform of a measure of bounded variation over $\mathbb{R}$. We then express the generalized analytic conditional Feynman integral of $G$ as two kinds of limits of nonconditional generalized Wiener integrals with a polygonal function and cylinder functions using a change-of-scale transformation. The choice of a complete orthonormal subset of $L_{2}[0, t]$ used in the transformation is independent of $e$.

Copyright 2016 by the Tusi Mathematical Research Group.

Received Aug. 25, 2015; Accepted Nov. 2, 2015.

2010 Mathematics Subject Classification. Primary 46T12; Secondary 28C20, 46 G12.

Keywords. analytic conditional Feynman integral, analytic conditional Wiener integral, conditional Wiener integral, Wiener integral, Wiener space. 


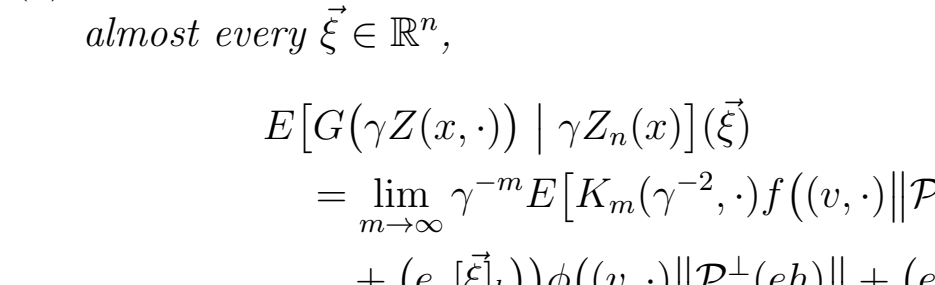

\title{
SCALE TRANSFORMATIONS FOR PRESENT POSITION-DEPENDENT CONDITIONAL EXPECTATIONS OVER CONTINUOUS PATHS
}

\author{
DONG HYUN CHO
}

Communicated by S. Barza

Abstract. Let $C[0, t]$ denote a generalized Wiener space, the space of realvalued continuous functions on the interval $[0, t]$, and define a random vector $Z_{n}: C[0, t] \rightarrow \mathbb{R}^{n}$ by

$$
Z_{n}(x)=\left(\int_{0}^{t_{1}} h(s) d x(s), \ldots, \int_{0}^{t_{n}} h(s) d x(s)\right)
$$

where $0<t_{1}<\cdots<t_{n}=t$ is a partition of $[0, t]$ and $h \in L_{2}[0, t]$ with $h \neq 0$ almost everywhere. Using a simple formula for a generalized conditional Wiener integral on $C[0, t]$ with the conditioning function $Z_{n}$, we evaluate the generalized analytic conditional Wiener and Feynman integrals of the cylinder function

$$
G(x)=f((e, x)) \phi((e, x))
$$

for $x \in C[0, t]$, where $f \in L_{p}(\mathbb{R})(1 \leq p \leq \infty)$, $e$ is a unit element in $L_{2}[0, t]$, and $\phi$ is the Fourier transform of a measure of bounded variation over $\mathbb{R}$. We then express the generalized analytic conditional Feynman integral of $G$ as two kinds of limits of nonconditional generalized Wiener integrals with a polygonal function and cylinder functions using a change-of-scale transformation. The choice of a complete orthonormal subset of $L_{2}[0, t]$ used in the transformation is independent of $e$.

Copyright 2016 by the Tusi Mathematical Research Group.

Received Aug. 25, 2015; Accepted Nov. 2, 2015.

2010 Mathematics Subject Classification. Primary 46T12; Secondary 28C20, 46 G12.

Keywords. analytic conditional Feynman integral, analytic conditional Wiener integral, conditional Wiener integral, Wiener integral, Wiener space. 


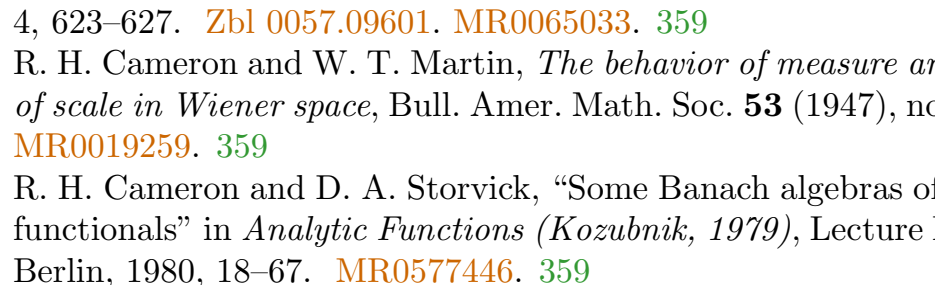

\title{
SCALE TRANSFORMATIONS FOR PRESENT POSITION-DEPENDENT CONDITIONAL EXPECTATIONS OVER CONTINUOUS PATHS
}

\author{
DONG HYUN CHO
}

Communicated by S. Barza

Abstract. Let $C[0, t]$ denote a generalized Wiener space, the space of realvalued continuous functions on the interval $[0, t]$, and define a random vector $Z_{n}: C[0, t] \rightarrow \mathbb{R}^{n}$ by

$$
Z_{n}(x)=\left(\int_{0}^{t_{1}} h(s) d x(s), \ldots, \int_{0}^{t_{n}} h(s) d x(s)\right)
$$

where $0<t_{1}<\cdots<t_{n}=t$ is a partition of $[0, t]$ and $h \in L_{2}[0, t]$ with $h \neq 0$ almost everywhere. Using a simple formula for a generalized conditional Wiener integral on $C[0, t]$ with the conditioning function $Z_{n}$, we evaluate the generalized analytic conditional Wiener and Feynman integrals of the cylinder function

$$
G(x)=f((e, x)) \phi((e, x))
$$

for $x \in C[0, t]$, where $f \in L_{p}(\mathbb{R})(1 \leq p \leq \infty)$, $e$ is a unit element in $L_{2}[0, t]$, and $\phi$ is the Fourier transform of a measure of bounded variation over $\mathbb{R}$. We then express the generalized analytic conditional Feynman integral of $G$ as two kinds of limits of nonconditional generalized Wiener integrals with a polygonal function and cylinder functions using a change-of-scale transformation. The choice of a complete orthonormal subset of $L_{2}[0, t]$ used in the transformation is independent of $e$.

Copyright 2016 by the Tusi Mathematical Research Group.

Received Aug. 25, 2015; Accepted Nov. 2, 2015.

2010 Mathematics Subject Classification. Primary 46T12; Secondary 28C20, 46 G12.

Keywords. analytic conditional Feynman integral, analytic conditional Wiener integral, conditional Wiener integral, Wiener integral, Wiener space. 